\title{
C \\ Artigo de revisão: a variabilidade da frequência cardíaca no desmame da ventilação mecânica
}

\section{ASSOBRAFIR \\ C I Ê N C I A}

\author{
Review article: heart rate variability in weaning from mechanical \\ ventilation
}

Renata Baltar da Silva* (1), Victor Ribeiro Neves² (10, Dário Celestino Sobral Filho (1)
'Programa de Pós-graduação em Ciências da Saúde, Universidade de Pernambuco (UPE), Recife, PE, Brasil

2Programa de Pós-graduação em Reabilitação e Desempenho Funcional, Universidade de Pernambuco (UPE), Campus Petrolina, Petrolina, PE, Brasil

\section{Como citar: Silva RB, Sobral Filho DC, Neves VR. Artigo de revisão: a variabilidade da frequência cardíaca no desmame da ventilação mecânica. ASSOBRAFIR Ciênc. 2022;13:e44284. https://doi. org/10.47066/2177-9333.AC.2020.0046}

Submissão em: Agosto 05, 2021

Aceito em: Janeiro 12, 2022

Estudo realizado em: Universidade de Pernambuco (UPE), Recife, PE, Brasil. Aprovação ética: Não se aplica.

*Autor correspondente: Renata Baltar da Silva. E-mail: renatabaltar@hotmail.com

\begin{abstract}
Resumo
Introdução: A ventilação mecânica (VM) é uma modalidade de suporte à vida na insuficiência respiratória. Quinze a 25 por cento dos pacientes apresentam dificuldades no desmame da VM. Alterações da variabilidade da frequência cardíaca (VFC) fornecem um indicador sensível e antecipado do comprometimento da saúde. Objetivo: Avaliar as evidências sobre o papel da VFC no desmame da VM. Métodos: Foi revisada a literatura através das bases de dados BVS, SciELO e PubMed, de estudos em humanos publicados até março de 2021, usando-se os descritores: ventilator weaning AND heart rate variability. Resultados: No PubMed, foramx encontrados vinte artigos e excluídos doze. Os nove trabalhos encontrados na BVS foram excluídos por duplicidade e na SciELO nenhum estudo foi encontrado. Assim, oito estudos fizeram parte da revisão com um total de 507 pacientes, com média de idade de 64,5 anos e a maioria do sexo masculino (61,5\%). A principal variável analisada nos estudos foi a VFC no domínio da frequência, porém medidas da VFC no domínio do tempo, medidas do fluxo respiratório, de sinais vitais e do status psíquico também foram utilizadas. Já o principal objetivo foi a VFC no desmame e sua associação ao desfecho. A VFC foi um preditor de arritmia cardíaca durante o desmame. A redução da VFC e a retirada da atividade vagal foram as principais alterações nos pacientes com falha no desmame. Conclusão: A VFC é uma ferramenta promissora para avaliação do desfecho do desmame da VM, visto que sua redução está associada à falha do desmame.
\end{abstract}

Palavras-chave: Ventilação Mecânica; Frequência Cardíaca; Extubação; Sistema Nervoso Autônomo; Terapia Intensiva.

\begin{abstract}
Background: Mechanical ventilation (MV) is a life support modality in respiratory failure. Fifteen to 25 percent of patients have difficulties in weaning from MV. Changes in heart rate variability (HRV) provide a sensitive and early indicator of health impairment. Aim: To assess the evidence on the role of HRV in weaning from MV. Methods: The literature was reviewed using the VHL, SciELO and PubMed databases of human studies published up to March 2021, using the descriptors: ventilator weaning AND heart rate variability. Results: In PubMed, twenty articles were found and twelve were excluded. The nine studies found in the VHL were excluded due to duplicity and in SciELO no studies were found. Thus eight studies were part of the review with a total of 507 patients, with a mean age of 64.5 years and most of them male (61.5\%). The main variable analyzed in the studies was the HRV in the frequency domain, however measures of the HRV in the time domain, measures of respiratory flow, vital signs and psychic status were also used. The main objective was HRV at weaning and its association with the outcome. HRV was a predictor of cardiac arrhythmia during weaning. The reduction in HRV and the withdrawal of vagal activity were the main changes in patients who failed to wean. Conclusion: HRV is a promising tool for evaluating the outcome of weaning from MV, since its reduction is associated with weaning failure.
\end{abstract}

Keywords: Mechanical Ventilation; Heart Rate; Extubation; Autonomic Nervous System; Intensive Care.

\section{INTRODUÇÃO}

A ventilação mecânica (VM) é uma modalidade crítica de suporte à vida em pacientes com insuficiência respiratória aguda (IRpA), entretanto, atrasos no processo de desmame da ventilação aumentam a mortalidade e os custos relacionados à unidade de terapia intensiva (UTI)1. Estima-se que $15 \%$ a $25 \%$ dos 
pacientes apresentam dificuldades durante o processo de desmame ${ }^{2}$. Diversos critérios objetivos e subjetivos têm sido utilizados como preditores fisiológicos para fornecer informações acerca do risco de falha do desmame, porém, procedimentos preditivos precisos ainda são incipientes ${ }^{3}$.

No Brasil, dados colhidos em 40 UTI's mostraram que $55,6 \%$ dos pacientes internados faziam uso de VM ${ }^{4}$. Embora a VM seja considerada o maior avanço no tratamento das IRpAs, ela pode gerar uma série de efeitos adversos relacionados à maior morbimortalidade de pacientes ${ }^{5,6}$. Logo, é essencial que o paciente seja retirado da VM o mais rápido quanto clinicamente possível ${ }^{7-9}$.

O teste de respiração espontânea (TRE) tem sido utilizado como método de avaliação do desmame da VM. Pacientes que realizam TRE com sucesso são considerados aptos para respirar espontaneamente e, então, submetidos à extubação orotraqueal7,10. A falha da extubação tem uma associação significativa independente com o aumento da mortalidade, além de outros efeitos adversos como maior tempo de hospitalização, custos mais elevados e maior necessidade de traqueostomia1,3,10.

A variabilidade da frequência cardíaca (VFC) é caracterizada por alterações periódicas e não-periódicas da frequência cardíaca (FC) e pode ser definida como um fenômeno fisiológico de variação no intervalo de tempo entre os batimentos cardíacos ${ }^{11,12}$. De modo geral, a VFC descreve as oscilações entre batimentos cardíacos consecutivos (intervalo R-R) e representa a modulação autonômica do coração ${ }^{13}$.

O sistema nervoso autônomo (SNA), através de suas terminações nervosas simpáticas e parassimpáticas (vagal), tem controle direto sobre a $\mathrm{FC}^{13,14}$. O aumento da atividade simpática e/ou inibição da atividade vagal repercute em aumento da FC, enquanto o aumento de atividade vagal implica em sua redução ${ }^{14,15}$.

A VFC é um fenômeno normal e esperado em indivíduos saudáveis e representa a capacidade do coração de responder a múltiplos estímulos fisiológicos e ambientais 13. Sabe-se que mudanças nos padrões da VFC fornecem um indicador sensível e antecipado de comprometimento da saúde. A redução da VFC é considerada um sinal de adaptação anormal e insuficiente do SNA e pode indicar a presença de mau funcionamento fisiológico do indivíduo ${ }^{16}$.

Assim, a VFC tem sido considerada um forte indicador de risco relacionado a eventos adversos em indivíduos normais e em pacientes com diversas doenças ${ }^{16}$. Em pacientes portadores de doenças cardiovasculares tem sido demonstrado que os índices de VFC encontramse reduzidos ${ }^{17,18}$. A redução da VFC está relacionada ao aumento da morbimortalidade cardiovascular ${ }^{19}$

Estudos apontam para a influência de alterações do sistema respiratório sobre a VFC. Foi observado que pacientes fumantes apresentam uma associação positiva entre o declínio da função pulmonar (menor pico de fluxo expiratório - PFE e volume expiratório forçado no primeiro segundo $-V_{E F}$ ) e a VFC reduzida ${ }^{20}$. Enquanto em indivíduos asmáticos, observou-se um desequilíbrio simpáticovagal com predomínio da atividade parassimpática e consequente redução da VFC ${ }^{21}$.

Diante da correlação observada entre a VFC e o binômio saúde-doença, assim como de sua interação com alterações do sistema respiratório, foi objetivo desta revisão avaliar as evidências científicas sobre o papel da VFC no desmame da VM existentes na literatura.

\section{MÉTODO}

Esta revisão foi realizada entre dezembro de 2020 e março de 2021. Avaliou estudos de diferentes delineamentos, disponíveis nas bases de dados Biblioteca Virtual em Saúde (BVS), Scientific Eletronic Library Online (SciELO) e U.S. National Library of Medicine and National Institutes of Health (PubMed); e que contivessem os descritores utilizados nesta revisão, que foram: ventilator weaning e heart rate variability, sendo o termo AND utilizado como operador booleano. O termo ventilator weaning foi selecionado mediante consulta ao Medical Subject Headings - MeSH, entretanto para o descritor heart rate variability não existe termo correspondente no MeSH.

A identificação e seleção dos estudos foi realizada por dois pesquisadores às cegas e considerou como critérios de inclusão estudos realizados em seres humanos que associassem a avaliação da VFC ao desmame da VM, publicados até março de 2021. Foram determinados requisitos metodológicos e populacionais, excluindose artigos de revisão, cartas aos editores, estudos realizados com pacientes menores de dezoito anos ou que apresentassem temas não pertinentes aos objetivos propostos.

A qualidade dos artigos foi descrita por meio do sistema Qualis/CAPES (Sistema Brasileiro de Avaliação dos Periódicos da Coordenação de Aperfeiçoamento de Pessoal de Nível Superior), em período de classificação do quadriênio 2013-2016. Para isso, analisou-se a classificação dos periódicos em que os artigos foram publicados, os quais são categorizados em estratos que variam de indicadores que vão de A1 (mais elevado) passando por A2, B1, B2, B3, B4, B5 e C (peso 0).

Foi considerado desmame da ventilação mecânica quando o paciente era submetido ao TRE ${ }^{6}$. Para isto, o paciente era colocado em Tubo em $T$ ou ventilação por pressão de suporte (PSV) de 5-7 $\mathrm{cmH}_{2} \mathrm{O}$ durante 30-120 minutos, e monitorizado para sinais de insucesso. Após o sucesso no TRE, seguido de extubação orotraqueal, foi considerado sucesso no desmame os pacientes que não necessitaram de reintubação orotraqueal nas primeiras 48 horas após a extubação.

A avaliação da VFC foi considerada nos estudos que realizaram análises pelos métodos no domínio do tempo e/ou no domínio da frequência por meio de registros eletrocardiográficos. 
O processo de seleção dos estudos ocorreu em três etapas. Na primeira foi realizada a leitura dos títulos e resumos dos artigos encontrados. Posteriormente, procedeu-se com a leitura dos textos dos manuscritos na íntegra e, por último, a identificação de possíveis artigos de interesse pela lista de referências. Além disso, artigos presentes em mais de uma das bases de dados tiveram as duplicatas excluídas.

\section{RESULTADOS}

Foram identificados 29 artigos a partir dos descritores utilizados nesta revisão. Na base de dados PubMed, foram encontrados 20; destes, 2 foram excluídos pela faixa etária da amostra estudada, 8 por não abordarem diretamente o tema de interesse e 2 por se tratar de cartas aos editores. $\mathrm{Na}$ base de dados BVS, foram encontrados 9 artigos, porém todos foram excluídos por duplicidades com os encontrados na PubMed. Na SciELO nenhum artigo foi encontrado. Assim, não foi incluído nenhum artigo da BVS, nem da SciELO.

Desta forma, do total de 29 artigos encontrados pelos descritores analisados, 21 foram excluídos, perfazendo uma amostra final de 8 artigos incluídos nesta revisão. A descrição do processo de seleção dos estudos encontrase sintetizada na Figura 1.

A Tabela 1 apresenta as principais características dos artigos selecionados, segundo o ano de publicação, local de realização da pesquisa, tamanho da amostra, objetivos, variáveis analisadas e conclusão dos estudos. Dos 8 trabalhos selecionados, 1 foi realizado no Brasil, 1 foi feito nos Estados Unidos da América (EUA), 3 na Europa e 3 em Taiwan. As publicações se concentram em periódicos da área médica, voltados para terapia intensiva, cardiologia, pneumologia e engenharia biomédica. O total de pacientes avaliados em todos os estudos foi 507, dos quais a maioria eram homens $(61,5 \%)$ e tinham média de idade de 64,5 anos. $O$ estudo de Orini et al. ${ }^{28}$ não descreveu a distribuição por sexo e a idade da amostra.

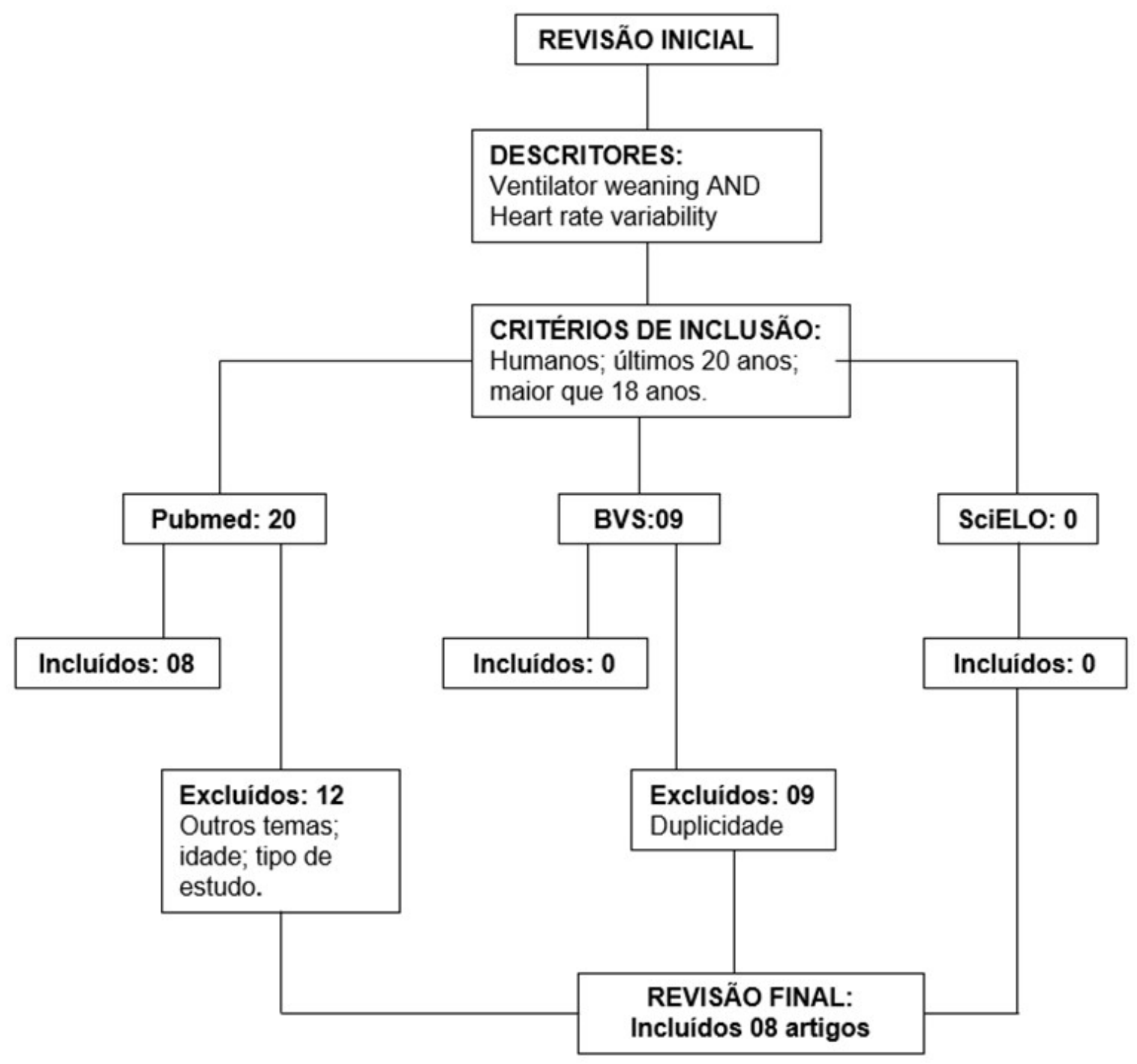

Figura 1. Fluxograma da seleção dos artigos para inclusão na revisão. 


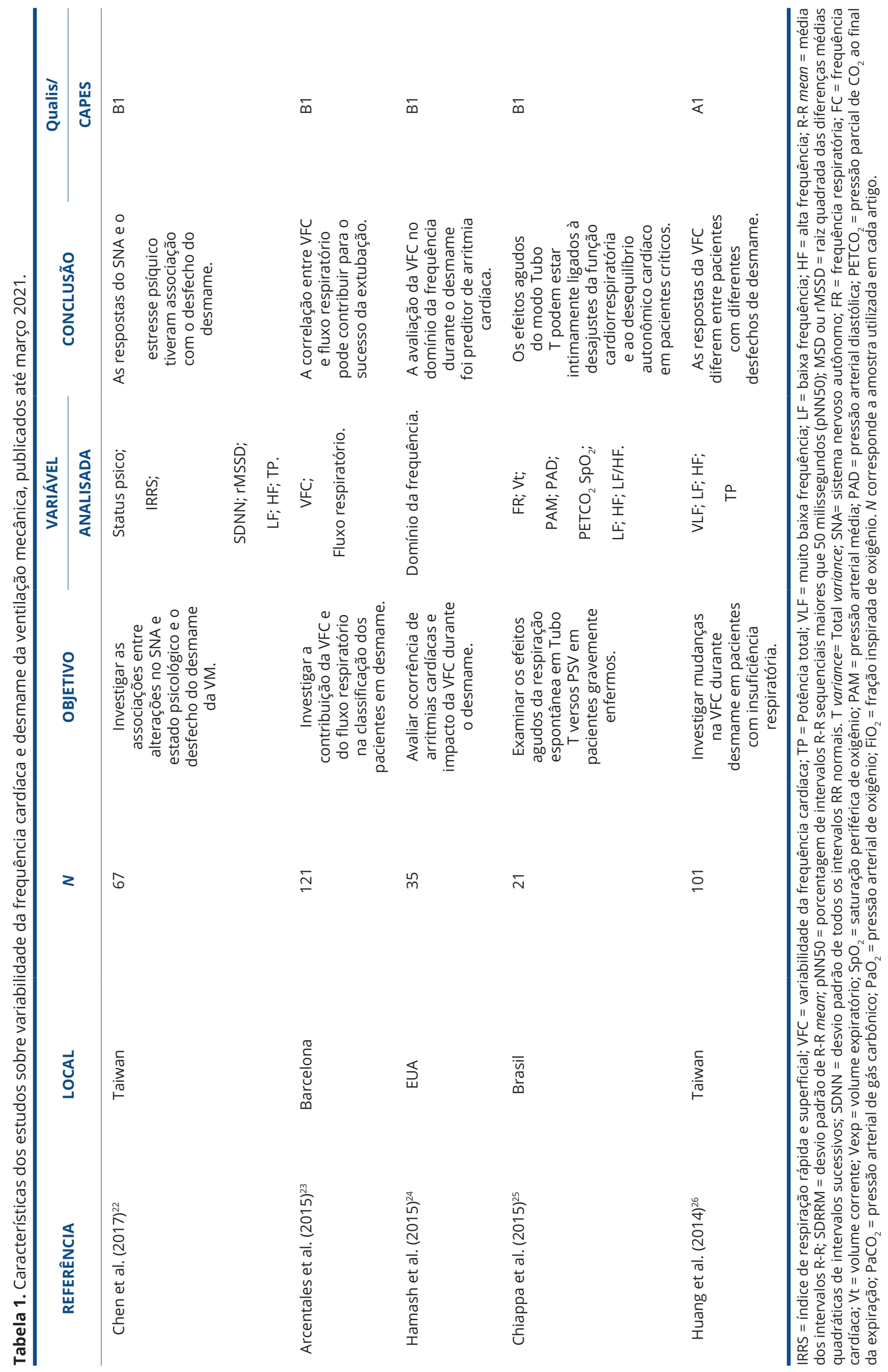




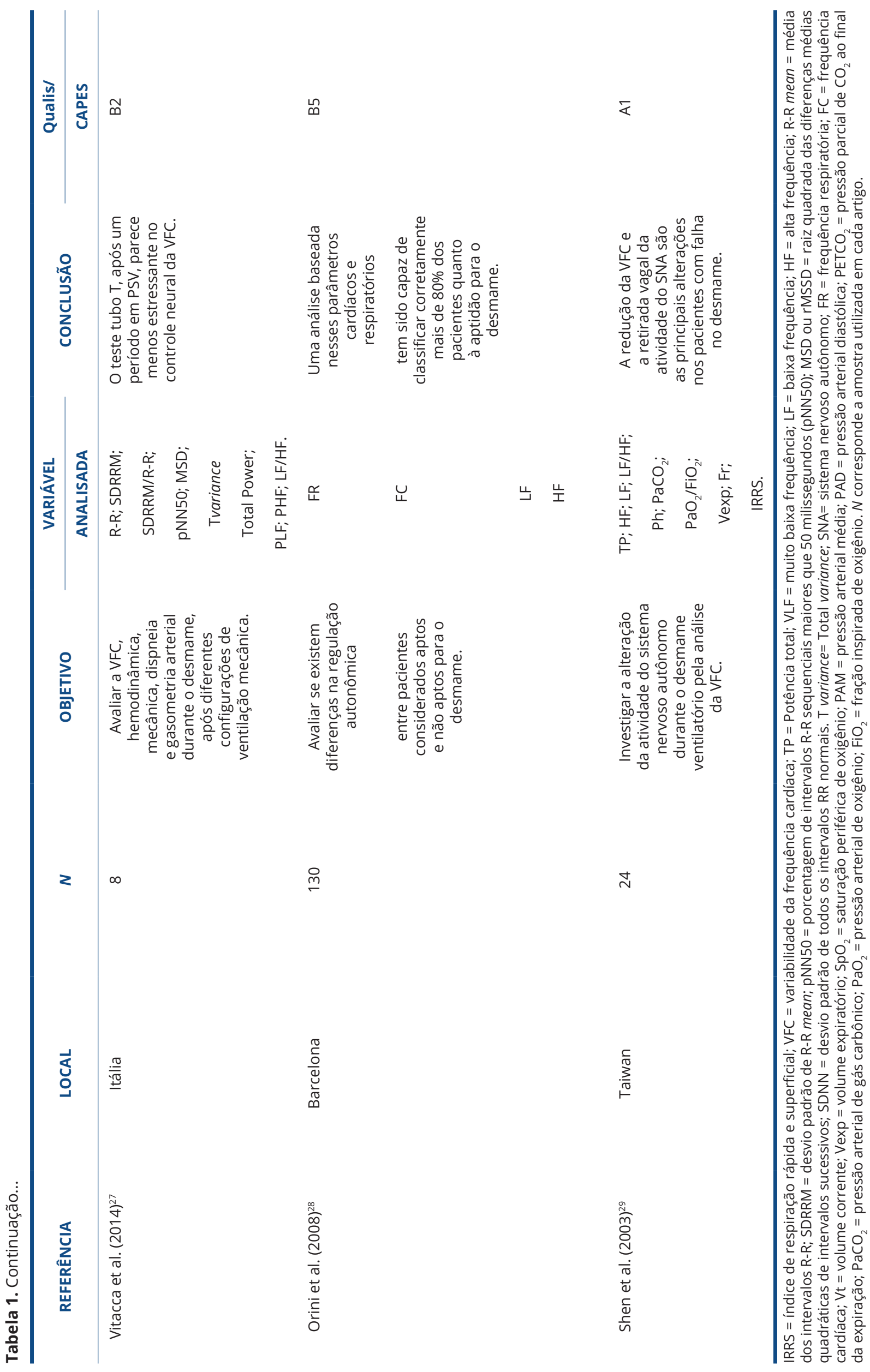


A análise da VFC no domínio da frequência foi realizada em todos os estudos incluídos, mensurando-se as variáveis: - Potência total (TP); - Muito baixa frequência (VLF); Baixa frequência (LF); Alta frequência (HF); relação LF/HF. Medidas da VFC no domínio do tempo foram utilizadas em 2 estudos, entre elas: média dos intervalos R-R (R-R mean); desvio padrão de R-R mean (SDRRM); coeficiente de variação SDRRM/R-R; porcentagem de intervalos $R-R$ sequenciais maiores que 50 milissegundos ( $p N N 50$ ); raiz quadrada das diferenças médias quadráticas de intervalos sucessivos (rMSSD); desvio padrão de todos os intervalos RR normais gravados, expresso em milissegundos (SDNN).

As demais variáveis analisadas em alguns estudos foram: variáveis respiratórias (Índice de respiração rápida e superficial - IRRS; sinal de fluxo respiratório; Volume corrente - Vt; volume expiratório - Vexp), medidas de sinais vitais (frequência respiratória - FR; pressão arterial média - PAM; pressão arterial diastólica - PAD; pressão parcial de $\mathrm{CO}_{2}$ no final da expiração - $\mathrm{PETCO}_{2}$; saturação periférica de oxigênio - $\mathrm{SpO}_{2}$ ), parâmetros gasométricos ( $\mathrm{PH}$; pressão arterial de $\mathrm{CO}_{2}-\mathrm{PaCO}_{2}$; relação pressão arterial de $\mathrm{O}_{2}$ e fração inspirada de $\mathrm{O}_{2}-\mathrm{PaO}_{2} / \mathrm{FiO}_{2}$ ) e status psíquico.

A maioria dos estudos objetivou analisar as alterações da VFC durante o processo de desmame e sua possível associação com o desfecho deste processo. A técnica de desmame mais frequente foi o TRE em Tubo T. Apenas 1 estudo realizou o TRE em PSV e 1 estudo realizou o TRE em modo ventilação mandatória intermitente sincronizada (SIMV), com redução gradual dos níveis pressóricos.

Foi observado que as respostas do SNA e o estresse psíquico tiveram associação com o desfecho do desmame. Pacientes com diferentes desfechos no desmame apresentam respostas diferentes da VFC. A avaliação da VFC durante o desmame foi considerada um preditor de arritmia cardíaca. Um estudo demonstrou que a avaliação da correlação entre a VFC e o sinal de fluxo respiratório pode contribuir para o sucesso da extubação.

Pacientes submetidos ao teste em Tubo T após uso de VM sem uso de pressão expiratória final positiva (PEEP) e com maiores pressões de suporte apresentaram maior estresse no controle neural da VFC. Os efeitos agudos do teste em Tubo $T$ podem estar intimamente ligados a desajustes da função cardiorrespiratória e ao desequilíbrio autonômico cardíaco em pacientes críticos. A redução da VFC e a retirada da atividade vagal do SNA foram as principais alterações nos pacientes com falha no desmame. Análises baseadas na VFC e parâmetros respiratórios são capazes de classificar corretamente mais de $80 \%$ dos pacientes quanto à aptidão para o desmame.

\section{DISCUSSÃO}

A ventilação mecânica é a intervenção de suporte à vida mais utilizada em pacientes gravemente enfermos. Após a resolução da doença de base e o sucesso no TRE, deve ser realizada a extubação orotraqueal o mais cedo possível.
De acordo com Lai et al., mesmo após extensiva avaliação, cerca de $10 \%$ a $19 \%$ dos pacientes falham na extubação, necessitam de reintubação e, consequentemente, aumentam a taxa de mortalidade ( $26 \%$ a $50 \%)^{23}$. Portanto, são necessárias novas estratégias que promovam maior sucesso no desmame e minimizem os danos.

O SNA é o corresponsável pela homeostasia do sistema cardiorrespiratório, por meio do balanço entre a atividade do sistema simpático e parassimpático, porém sua monitorização não é comumente utilizada na prática diária das unidades de cuidados intensivos ${ }^{30}$. A VFC tem sido utilizada como ferramenta para avaliar o estado de saúde de indivíduos em diferentes circunstâncias e, cada vez mais, tem sido caracterizada a relação direta entre a habilidade de adaptação do organismo a diferentes situações e o binômio saúde-doença ${ }^{31}$.

Nesta revisão, foi observado que as medidas de VFC no domínio da frequência foram utilizadas em todos os artigos. Atribuímos isto ao fato de a VFC no domínio da frequência, denominada análise espectral, permitir decompor o sinal eletrocardiográfico em diferentes componentes de frequência e, assim, determinar a participação individual de cada uma das divisões do SNA (simpático e parassimpático) em diferentes situações fisiológicas e patológicas sendo mais apropriada para avaliações de curto prazo ${ }^{12}$.

Além disso, é possível identificar a relação de cada banda de frequência com os principais sistemas que interferem na VFC (sistemas respiratório, vasomotor, termorregulador, da renina-angiotensina e sistema nervoso central) permitindo melhor estudá-los. Dois estudos utilizaram a análise da VFC no domínio do tempo, porém, essas medidas representam a variabilidade global e refletem atividade simpática e parassimpática. Análises no domínio da frequência permitem individualizar a participação de cada divisão do SNA (simpático e parassimpático) em diferentes situações fisiológicas e patológicas, assim como sua relação com os principais sistemas que interferem na VFC (sistema respiratório, vasomotor, termorregulador etc. $)^{12}$.

As principais variáveis respiratórias estudadas foram IRRS, sinais vitais e parâmetros gasométricos. Lai et al. ${ }^{32}$ observaram que o IRRS é um fator de risco independente para o sucesso da extubação em paciente após um TRE bem sucedido e é considerado o índice preditivo com maior acurácia ${ }^{7,32}$. Diversos estudos utilizaram medidas de fluxo respiratório, Vt, Vexp, assim como parâmetros gasométricos, a fim de prever o desfecho da extubação. Porém, o pequeno tamanho das amostras utilizadas não permite considerar os resultados precisos, nem os extrapolar para a população em gera| ${ }^{32-34}$.

A maioria dos estudos objetivou analisar a correlação entre a VFC e o desfecho do desmame. A técnica de desmame mais frequente foi o TRE em Tubo T. Apenas um estudo realizou o TRE em PSV e um estudo realizou o TRE em modo SIMV, com redução gradual dos níveis 
pressóricos. De acordo com diretrizes brasileiras de ventilação mecânica (2014), durante o TRE, o paciente deve ser colocado em Tubo em T ou PSV de 5-7 cmH2O durante 30-120 minutos e ser monitorizado para sinais de insucesso ${ }^{7}$.

Segundo Barbas7, a técnica de desmame em SIMV deve ser evitada visto que pode prolongar o tempo de VM e seus efeitos adversos. Quanto a comparação de eficácia dos testes em PSV e em Tubo T, não existe um consenso na literatura. Diversos autores sugerem que ambos os testes são equivalentes, não havendo vantagem de um sobre o outro ${ }^{35,36}$. Em contrapartida, Colombo et al. ${ }^{37}$ e Serra et al. ${ }^{38}$ observaram que a técnica PSV estava associada a um menor tempo de VM.

Huang et al. ${ }^{26}$, no maior estudo já realizado sobre a VFC e os resultados do TRE, observaram que pacientes com diferentes desfechos no desmame apresentam respostas diferentes da VFC.

Nesse estudo, a redução da VFC foi significativamente associada com a falha do TRE e a incapacidade de aumentar a VFC após a extubação foi correlacionada com a falha da extubação mesmo em pacientes que tiveram sucesso no TRE. Assim, monitorar a VFC durante o processo de desmame pode ajudar a prever o desfecho e melhorar os resultados ${ }^{26}$. Em concordância, Shen et al. ${ }^{29}$ já haviam observado que a redução da VFC e a supressão da atividade vagal são as principais alterações nos pacientes com insucesso no desmame.

Um estudo demonstrou que a avaliação da correlação entre a VFC e o sinal de fluxo respiratório pode contribuir para o sucesso da extubação. Segundo Arcentales et al., a análise da VFC isolada pode predizer os sujeitos que terão sucesso na extubação. Entretanto, a associação da análise do fluxo respiratório pode auxiliar a distinguir, entre aqueles que falham na extubação, os que não tem sucesso no TRE, daqueles que após a extubação necessitam ser reintubados nas próximas $48 \mathrm{hs}^{23}$.

Sabe-se que, embora a taxa de sucesso na extubação esteja aumentando nos últimos anos, o percentual de reintubação permanece similar, variando de 6\% a 25\% em diferentes populações ${ }^{39}$. Assim os resultados do estudo de Arcentales tornam-se bastante relevantes, visto que a reintubação aumenta a mortalidade e um índice preditivo de maior acurácia poderia evitá-la.

Outros autores analisaram a VFC e parâmetros respiratórios associados e sugeriram que esta análise combinada é capaz de classificar corretamente mais de $80 \%$ dos pacientes quanto à aptidão para o desmame. Eles sugerem que existem diferenças importantes na regulação do SNA entre pacientes considerados prontos para respiração espontânea e pacientes que não estão ${ }^{28}$.

\section{CONCLUSÃO}

Os estudos demonstraram que a VFC apresenta resultados que estão associados inversamente à gravidade clínica e ao prognóstico de doentes críticos podendo ser uma ferramenta promissora para avaliação do desfecho do desmame da VM, visto que reduções da VFC estão relacionadas ao seu insucesso. Entretanto, existe uma limitação de seu uso como ferramenta de prognóstico e consequente dificuldade de introduzir sua monitorização na prática diária das unidades de cuidados intensivos. Mais estudos são necessários para que sejam encontrados índices preditivos mais acurados e, desta forma, melhorar o desfecho do desmame e reduzir a mortalidade dos pacientes.

\section{FONTE DE FINANCIAMENTO}

Nada a declarar.

\section{CONFLITO DE INTERESSES}

Nada a declarar.

\section{AGRADECIMENTOS}

O presente trabalho foi realizado com apoio da Coordenação de Aperfeiçoamento de Pessoal de Nível Superior - Brasil (CAPES) - Código de Financiamento 001.

\section{REFERÊNCIAS}

1. Macintyre NR, Cook DJ, Ely EW Jr, Epstein SK, Fink JB, Heffner JE, et al. Evidence-based guidelines for weaning and discontinuing ventilatory support: a collective task force facilitated by the American College of Chest Physicians; the American Association for Respiratory Care; and the American College of Critical Care Medicine. Chest. 2001;120(6, Suppl):375S-95S. http://dx.doi.org/10.1378/ chest.120.6_suppl.375S. PMid:11742959.

2. Thille AW, Boissier F, Ben-Ghezala H, Razazi K, MekontsoDessap A, Brun-Buisson C, et al. Easily identified at-risk patients for extubation failure may benefit from noninvasive ventilation: a prospective before-after study. Crit Care. 2016 Fev;20(48):1-8. http://dx.doi.org/10.1186/s13054-016-12282. PMid:26926168.

3. Teixeira C, Maccari JG, Vieira SRR, Oliveira RP, Savi A, Machado ASA, et al. Impacto de um protocolo de desmame de ventilação mecânica na taxa de falha de extubação em pacientes de difícil desmame. J Bras Pneumol. 2012 Jun;38(3):364-71. http://dx.doi.org/10.1590/S180637132012000300012 . PMid:22782607.

4. Damasceno MPCD, David CMN, Souza PCSP, Chiavone PA, Cardoso LTQ, Amaral JLG, et al. Ventilação Mecânica no Brasil. Aspectos Epidemiológicos. Rev Bras Ter Intensiva. 2006;18(3):219-28. http://dx.doi.org/10.1590/S0103507X2006000300002. PMid:25310434.

5. Dasta JF, Mclaughlin TP, Mody SH, Piech CT. Daily cost of an intensive care unit day: the contribution of mechanical ventilation. Crit Care Med. 2005;33(6):1266-71. 
http://dx.doi.org/10.1097/01.CCM.0000164543.14619.00. PMid:15942342.

6. Carvalho CRR. Ventilator-associated pneumonia. J Bras Pneumol. 2006;32(4):xx-xxii. http://dx.doi.org/10.1590/ S1806-37132006001100003. PMid:17278314.

7. Barbas CS, Ísola AM, Farias AM, Cavalcanti AB, Gama AM, Duarte AC, et al. Brazilian recommendations of mechanical ventilation 2013. Part 2. Rev Bras Ter Intensiva. 2014 JulSet;26(3):215-39. PMid:25295817.

8. Brochard L, Rauss A, Benito S, Conti G, Mancebo J, Rekik $\mathrm{N}$, et al. Comparison of three methods of gradual withdrawal from ventilatory support during weaning from mechanical ventilation. Am J Respir Crit Care Med. 1994;150(4):896903. http://dx.doi.org/10.1164/ajrccm.150.4.7921460. PMid:7921460.

9. Esteban A, Frutos F, Tobin MJ, Alía I, Solsona JF, Valverdú I, et al. A comparison of four methods of weaning patients from mechanical ventilation. N Engl J Med. 1995;332(6):34550. http://dx.doi.org/10.1056/NEJM199502093320601. PMid:7823995.

10. Epstein SK. Weaning from ventilatory support. Curr Opin Crit Care. 2009;15(1):36-43. http://dx.doi.org/10.1097/ MCC.0b013e3283220e07. PMid:19179869.

11. Chuduc H, Nguyenphan K, Nguyenviet D. A review of heart rate variability and its applications. APCBEE Procedia. 2013;7:805. http://dx.doi.org/10.1016/j.apcbee.2013.08.016.

12. RASSI AJR. Compreendendo melhor as medidas de análise da variabilidade da frequência cardíaca. J Diag Cardiol. 2000;8.

13. Vanderlei LC, Pastre CM, Hoshi RA, Carvalho TD, Godoy MF. Basic notions of heart rate variability and its clinical applicability. Rev Bras Cir Cardiovasc. 2009 Abr-Jun;24(2):205-17. http://dx.doi.org/10.1590/S010276382009000200018. PMid:19768301.

14. Aubert $A E$, Seps $B$, Beckers F. Heart rate variability in athletes. Sports Med. 2003;33(12):889-919. http://dx.doi. org/10.2165/00007256-200333120-00003. PMid:12974657.

15. Rajendra Acharya U, Paul Joseph K, Kannathal N, Lim CM, SuriJS. Heart rate variability: A review. Med Biol Eng Comput. 2006;44(12):1031-51. http://dx.doi.org/10.1007/s11517-0060119-0. PMid:17111118.

16. Pumprla J, Howorka K, Groves D, Chester M, Nolan J. Functional assessment of heart rate variability : physiological basis and practical applications. Int J Cardiol. 2002;84(1):1 14. http://dx.doi.org/10.1016/S0167-5273(02)00057-8. PMid:12104056.

17. Karas $M$, Larochelle $P$, LeBlanc RA, Dubé B, Nadeau R, Champlain Jd. Attenuation of autonomic nervous system functions in hypertensive patients at rest and during orthostatic stimulation. J Clin Hypertens (Greenwich). 2008 Feb;10(2):97-104. http://dx.doi.org/10.1111/j.17517176.2008.07324.x. PMID: 18256574.

18. Terathongkum S, Pickler RH. Relationships among heart rate variability, hypertension, and relaxation techniques. J Vasc Nurs. 2004;22(3):78-82, quiz 83-4. http://dx.doi. org/10.1016/j.jvn.2004.06.003. PMid:15371972.

19. Thayer JF, Yamamoto SS, Brosschot JF. The relationship of autonomic imbalance, heart rate variability and cardiovascular disease risk factors. Int J Cardiol. 2010;141(2):122-31. http:// dx.doi.org/10.1016/j.ijcard.2009.09.543. PMid:19910061.

20. Behera JK, Kumar N, Sharma K, Mishra R, Roy PS, Sood S. Heart rate variability and its correlation with pulmonary function test of smokers. Heart Views. 2013;14(1):22-5. http://dx.doi. org/10.4103/1995-705X.107116.

21. Gupta J, Dube A, Singh V, Gupta RC. Spectral analysis of heart rate variability in bronchial asthma patients. Indian J Physiol Pharmacol. 2012;56(4):330-6. PMid:23781652.

22. Chen Y-J, Hwang S-L, Li C-R, Yang C-C, Huang K-L, Lin $C-Y$, et al. Vagal withdrawal and psychological distress during ventilator weaning and the related outcomes. J Psychosom Res. 2017;101:10-6. http://dx.doi.org/10.1016/j. jpsychores.2017.07.012. PMid:28867413.

23. Arcentales A, Caminal P, Diaz I, Benito S, Giraldo BF. Classification of patients undergoing weaning from mechanical ventilation using the coherence between heart rate variability and respiratory flow signal. Physiol Meas. 2015;36(7):1439-52. http://dx.doi.org/10.1088/09673334/36/7/1439. PMid:26020593.

24. Hammash MH, Moser DK, Frazier SK, Lennie TA, HardinPierce M. Heart variability as a predictor of cardic dysrhthmias during weaning from mechanical ventilation. Am J Crit Care. 2015;24(2):118-27. http://dx.doi.org/10.4037/ ajcc2015318. PMid:25727271.

25. Güntzel Chiappa AM, Chiappa GR, Cipriano G Jr, Moraes RS, Ferlin EL, Borghi-Silva A, et al. Spontaneous breathing trial in T-tube negatively impact on autonomic modulation of heart rate compared with pressure support in critically ill patients. Clin Respir J. 2017;11(4):489-95. http://dx.doi.org/10.1111/ crj.12363. PMid:26269215.

26. Huang C-T, Tsai Y-J, Lin J-W, Ruan S-Y, Wu H-D, Yu C-J. Application of heart-rate variability in patients undergoing weaning from mechanical ventilation. Crit Care. 2014;18(1):R21. http://dx.doi.org/10.1186/cc13705. PMid:24456585.

27. Vitacca M, Scalvini S, Volterrani M, Clini EM, Paneroni M, Giordano A, et al. In COPD patients on prolonged mechanical ventilation heart rate variability during the T-piece trial is better after pressure support plus PEEP: a pilot physiological study. Heart Lung. 2014;43(5):420-6. http://dx.doi. org/10.1016/j.hrtlng.2014.04.003. PMid:24856229.

28. Orini M, Giraldo BF, Bailón R, Vallverdu M, Mainardi L, Benito $\mathrm{S}$, et al. Time-frequency analysis of cardiac and respiratory parameters for the prediction of ventilator weaning. Annu Int Conf IEEE Eng Med Biol Soc. 2008;2008:2793-6. http:// dx.doi.org/10.1109/IEMBS.2008.4649782. PMid:19163285.

29. Shen H-N, Lin L-Y, Chen K-Y, Kuo P-H, Yu C-J, Wu H-D, et al. Changes of heart rate variability during ventilator weaning. Chest. 2003;123(4):1222-8. http://dx.doi.org/10.1378/ chest.123.4.1222. PMid:12684315.

30. Bento L, Fonseca-Pinto R, Póvoa P. Autonomic nervous system monitoring in intensive care as a prognostic tool. Systematic review. Rev Bras Ter Intensiva. 2017;29(4):4819. http://dx.doi.org/10.5935/0103-507X.20170072. PMid:29340538.

31. Selig FA, Tonolli ER, Silva EV, Godoy MF. Heart rate variability in preterm and term neonates. Arq Bras Cardiol. 2011 Jun;96(6):443-9. http://dx.doi.org/10.1590/S0066782X2011005000059. PMid:21584479.

32. Lai C-C, Chen C-M, Chiang S-R, Liu W-L, Weng S-F, Sung $M-I$, et al. Establishing predictors for successfully planned endotracheal extubation. Medicine (Baltimore). 2016;95(41):e4852. http://dx.doi.org/10.1097/ MD.0000000000004852. PMid:27741103.

33. Menon N, Joffe AM, Deem S, Yanez ND, Grabinsky $A$, Dagal $A H$, et al. Occurrence and complications of 
tracheal reintubation in critically ill adults. Respir Care. 2012;57(10):1555-63. http://dx.doi.org/10.4187/ respcare.01617. PMid:22324979.

34. Liu Y, Wei L-Q, Li G-Q, Lv F-Y, Wang H, Zhang Y-H, et al. A decision-tree model for predicting extubation outcome in elderly patients after a successful spontaneous breathing trial. Anesth Analg. 2010;111(5):1211-8. http://dx.doi. org/10.1213/ANE.0b013e3181f4e82e. PMid:20841406.

35. Costa AD, Rieder MM, Vieira SRR. Desmame da Ventilação Mecânica Utilizando Pressão de Suporte ou Tubo T. Comparação entre Pacientes Cardiopatas e não Cardiopatas. Arq Bras Cardiol. 2005;85(1):32-8. http://dx.doi.org/10.1590/ S0066-782X2005001400007. PMid:16041452.

36. José A, Oliveira LRC, Dias ECP, Fuin DB, Leite LG, Guerra GS, et al. Ventilação mecânica não invasiva aplicada em pacientes com insuficiência respiratória aguda após extubação traqueal. Rev Bras Ter Intensiva. 2006;18(4):338-
43. http://dx.doi.org/10.1590/S0103-507X2006000400004. PMid:25310546.

37. Colombo T, Boldrini AF, Juliano SRR, Juliano MCR, Houly JGS, Gebara OCE, et al. "Implementação, avaliação e comparação dos protocolos de desmame com Tubo-T e Pressão Suporte associada à pressão expiratória final positiva em pacientes submetidos à ventilação mecânica por mais de 48 horas em unidade de terapia intensiva. Rev Bras Ter Intensiva. 2007;19(1):31-7. PMid:25310657.

38. Serra MP, Carr AMG, Cardoso FEF, Torquato JA. Comparative study between the techniques: $t$ tube and pressure support in weaning from mechanical ventilation. Rev Saúde. 2011;5(4):32-9.

39. Esteban A, Frutos-Vivar F, Muriel A, Ferguson ND, Peñuelas $\mathrm{O}$, Abraira $\mathrm{V}$, et al. Evolution of mortality over time in patients receiving mechanical ventilation. Am J Respir Crit Care Med. 2013;188(2):220-30. http://dx.doi.org/10.1164/rccm.20121221690 C. PMid:23631814. 